Адрес статьи / To link this article: http://cat.ifmo.ru/ru/2018/v3-i4/152

\title{
Проблема классификации компьютерных игр в контексте психологических исследований геймеров
}

\author{
Н.В. Богачева ${ }^{1}$, А.Е. Войскунский ${ }^{2}$ \\ ${ }^{1}$ ФГАОУ ВО Первый Московский государственный медицинский университет имени И.М. Сеченова \\ Министерства здравоохранения Российской Федерации (Сеченовский Университет), Россия \\ bogacheva. nataly@gmail.com \\ ${ }^{2}$ ФГБОУ ВО Московский государственный университет имени М.В. Ломоносова, Россия

$$
\text { vae-msu@mail.ru }
$$

\begin{abstract}
Аннотация. Статья посвящена рассмотрению основных подходов к классификации компьютерных игр, применяемых в психологии и смежных науках. Классификации компьютерных игр рассматриваются с точки зрения их реального применения для проведения психологических исследований, с указанием их потенциальных достоинств и недостатков. Подробно анализируется т.н. жанровая классификация компьютерных игр, ее варианты и критика со стороны ученых; анализируются основные направления психологических исследований, опирающихся на представление об игровых жанрах. Наряду с жанровым делением компьютерных игр, а также более простыми критериями классификации (по содержанию игры, количеству игроков, типу игры - онлайн или офлайн), анализируются классификации компьютерных игр, построенные по психологическим основаниям - ведущей игровой мотивации, приобретаемым в игре навыкам, ролевой позиции игрока. Представлен обзор исследований демонстрируется неоднородность психологических особенностей игроков в компьютерные игры («геймеров») в зависимости от их игровых предпочтений. Эмпирическая часть работы описывает исследование когнитивно-стилевых и личностных (эмпатия, импульсивность, склонность к риску) особенностей игроков, предпочитающих онлайн и офлайн игры $(\mathrm{n}=223)$. Получены результаты, демонстрирующие выраженную когнитивностилевую (высокая поленезависимость, низкая импульсивность) специфику игроков в офлайн игры, а также высокую склонность к риску, характерную для онлайн игроков. Интерпретация результатов дает с позиции описанной в статье специфики разных форм компьютерной игровой деятельности.
\end{abstract}

Ключевые слова: компьютерная игра, геймеры, классификация компьютерных игр, онлайн игры, офлайн игры, импульсивность, когнитивные стили, полезависимость, киберспорт

\section{1. Введение}

Компьютерные игры являются одним из популярнейших увлечений современных детей, подростков и молодежи в целом. Готовность активных компьютерных игроков («геймеров») посвящать игре десятки часов в неделю нередко становится поводом для беспокойства родных, друзей, педагогов, психологов и врачей. Одновременно с этим, перед учеными встает вопрос о 
потенциальных причинах подобного увлечения, а также о его возможных последствиях для психики игроков, как позитивных, так и негативных. Направления психологических исследований геймеров, сформулированные еще в 80-х гг. ХX века, актуальны и поныне. Среди них: возникновение зависимости от видеоигр [1], развитие рабочей памяти, зрительного внимания, стратегического и логического мышления, навыков принятия решений $[1,2]$, зрительно-моторной координации, пространственного мышления $[1,3]$, эмоциональной и мотивационной специфики [4], появление новых форм идентичности у детей и подростков, развитие «компьютерной культуры» в целом [1]. Уже в 1998 г. в сборнике статей американских авторов, посвященном анализу возрастных, культурологических, гендерных, социологических, психологических, футурологических аспектов детской игры и претендующем на определенную энциклопедичность, в нескольких работах рассматривалось воздействие видеоигр, в том числе - коллективных - на психическое развитие ребенка [5]. К настоящему времени фронт исследований расширился, в том числе и за счет более активного изучения взрослых геймеров.

Несмотря на накопленные за более чем тридцатилетнюю историю эмпирических исследований данные, единое представление о личностной и когнитивной специфике игроков в компьютерные игры все еще сформировано; результаты зачастую противоречивы и неоднозначны. Так, например, игроки в компьютерные игры оцениваются одними авторами как импульсивные и склонные принимать решения методом проб и ошибок [6, 7], в других же исследованиях показаны как характеризующиеся низкой импульсивностью, ригидные $[8,9]$. Противоречивыми остаются и оценки связи компьютерных игр с элементами насилии и агрессией, проявляемой (или не проявляемой) геймерами в реальной жизни [10-12]. В последнее время возрастает количество исследований игроков в компьютерные игры с использованием методов нейропсихологии, но и они пока не позволяют получить непротиворечивые результаты [см. напр. 13, 14]. В то же время, все более активное применение игровых технологий для обучения, а также превращение в ряде стран компьютерных игр в спортивную дисциплину усиливает интерес к данной проблематике.

Представляется, что одной из причин возникновения противоречий между результатами различных исследований является внутренняя неоднородность такого явления как компьютерная игровая деятельность. Современные компьютерные игры весьма разнообразны по своей форме, содержанию, задачам, которые ставятся перед игроками. В свою очередь, игроки, предпочитающие разные игры или даже стили прохождения одной и той же игры, также характеризуются спецификой мотивации [15], различиями в интенсивности игры, что может определять возникновение той или иной когнитивной специфики [16]; наличием или отсутствием игровой зависимости [напр., 9]. Иными словами, рассмотрение геймеров как единой группы, характеризующейся сходными когнитивными и личностными особенностями методологически неверно. Напротив, при анализе и интерпретации данных эмпирических исследований необходимо обращать внимание на то, о каких именно играх и каких игроках идет речь. Однако, последнее дополнительно осложняется отсутствием единого представления о классификации компьютерных игр в психологии и других науках.

\section{2. Проблема классификации компьютерных игр}

В 2014 году пользователи Интернета попытались составить исчерпывающий список, который включал бы все когда-либо выходившие видеоигры (для любых игровых устройств, включая игровые автоматы, приставки, компьютеры). Согласно собранными данным, на тот момент в мире насчитывалось от 43 до более чем 90 тысяч игровых наименований [17, 18]. В 2016 году число наименований компьютерных игр, распространяемых только одним из нескольких популярных цифровых сервисов по их продаже, превысило 10 тысяч [19]. Очевидно, что подобное многообразие, проявляющееся в виде различных игровых сюжетов, визуальнографических элементов, технических средств, игровых механик, задач, которые ставятся перед игроком и т.П., обосновывает потребность в той или иной систематизации компьютерных игр.

В наиболее общем виде эффективная классификация компьютерных игр должна решать следующие задачи: идентификацию и группировку игр по наблюдаемым, объективным основаниям; соотнесение и поиск похожих по своим характеристикам игр для удобства пользователей; осуществление эффективной рекламы коммерческих игр; облегчение обучения и проведения исследований [20]. Одним их традиционных критериев классификации компьютерных

Культура и технологии, Том 3, № 4 
игр, понятным как разработчикам и игрокам, так и ученым, является «жанр» игры [21]. При этом, в отличие от литературы и искусства, где понятие «жанр» связано со стилевыми особенностями, сюжетом, темой произведения, а также эмоциями, которые оно призвано вызывать, при выделении «жанров» компьютерной игры, как правило, в первую очередь оценивается характер действий игрока, а также интерактивность виртуального мира игры, в то время как нарративные элементы, общая тематика игры и визуальные оставляющие оказываются вторичными при отнесении игры к той или иной группе [20, 22]. Один из вариантов жанровой классификации компьютерных игр, предложенный в работе А.А. Аветисовой [21], включает следующие категории игр и их критерии:

- «экшн»-игры (от англ. action - действие), подвидами которых являются «шутеры» (игры-«стрелялки», от англ. to shoot - стрелять) и «файтинги» (игры-«драки», от англ. to fight - сражаться). Игры этого жанра предполагают активное преодоление препятствий и уничтожение противников, в качестве которых могут выступать как виртуальные персонажи, так и персонажи, управляемые другими игроками. Зачастую игры этого жанра предъявляют высокие требования к зрительно-моторной координации, скорости реакции;

- «аркадbl» - игры с примитивными сценарием и системой управления, требующие максимально высокой скорости реакции и быстрого принятия решений;

- «квесты» (от англ. quest - поиск) - игры, обладающие сложным повествовательным сюжетом, продвижение по которому достигается путем решения головоломок, поиска предметов и способов их применения;

- «симуляторы» - игры, имитирующие какую-либо деятельность или область реальности: управление транспортным средством, спортивной командой, жизнью человека. Многие из них характеризуются стремлением к максимально точному воспроизведению физических характеристик реальности;

- «ролевые игры» (РПГ, RPG, от англ. role-play games) - игры с развернутой сюжетной составляющей, в которых игроку предлагается взять на себя роль того или иного персонажа. С точки зрения игровых действий РПГ-игры могут включать в себя элементы как жанра «экшн», так и сложные головоломки, однако предполагают обязательное развитие персонажа в плане игровых навыков или «характера» персонажа (за счет принимаемых игроком решений, как поступить в той или иной ситуации);

- «стратегии» - игры с военным или экономическим сюжетом, предполагающие выработку определенной стратегии и тактики. Подразделяются на игры в реальном времени (когда все решения необходимо принимать здесь и сейчас, в постоянно меняющихся динамических условиях) и пошаговые (когда игроки или компьютерные противники действуют по очереди, что позволяет обдумать следующий ход, однако подразумевает ограничения действий, которые могут быть совершены за один раз);

- «mрадищионные игры» - виртуальные аналоги реальных игр, например, шахмат.

Жанровые классификации компьютерных игр, подобные приведенной выше, широко применяются психологами при проведении эмпирических исследований. Некоторые авторы предпочитают изучать отдельные жанры: например, развитие у геймеров селективности зрительного внимания и пространственных способностей нередко демонстрируются на материале компьютерных игр жанра «экшн», в силу их динамичности, сложности и высокой мотивационной привлекательности [23, 24]. Развитие других когнитивных функций, например, когнитивной гибкости, напротив, связывают с опытом игры в стратегические игры [25]. Сравнительные исследования геймеров, предпочитающих разные игровые жанры, нередко также позволяют выявить психологическое своеобразие. Так, продемонстрирована повышенная импульсивность игроков в компьютерные игры жанра «шутер» по сравнению с игроками, предпочитающими стратегии [7]. Нейропсихологи также выявили различия в объеме зон мозга, отвечающих за пространственное мышление у игроков в разные игровые жанры: у геймеров, предпочитающих логические и аркадные игры, объем серого вещества в этих зона повышен (и может увеличиваться в ходе специально организованных сеансов игры [13]), в то время как у игроков в игры «экшн»,

International Culture \& Technology Studies, Vol. 3, No. 4 
наоборот, снижен [26]. Другое исследование показывает, что даже при изучении одного и того же игрового жанра («шутеры» от первого лица), изменения в объеме серого вещества гиппокампа будут различаться для игроков, использующих разные стратегии ориентации в игровом пространстве, причем значимые изменения достигаются уже при 90-то часовом опыте игры [14].

Таким образом, применение жанрового критерия для классификации компьютерных игр в психологии представляется оправданным в том числе с точки зрения эмпирических исследований. Наряду с относительной простотой применения (все существующие игры, так или иначе, уже отнесены к определенным жанрам), к достоинствам жанровых классификаций можно отнести их универсальность и понятность не только для психологов, так и для программистов, и рядовых игроков, а также потенциальную возможность адаптировать такие классификации под новые игры путем выделения новых жанров и их подвидов. Однако уже сейчас жанровые классификации компьютерных игр сталкиваются с рядом трудностей и рассматриваются некоторыми авторами как устаревшие [20]. Несмотря на относительно сходные основания, жанровые классификации компьютерных игр довольно разнообразны. Так, M. Wolf выделяет 42 игровых жанра (и это без учета многопользовательских онлайн игр, набравших популярность уже после создания этой классификации) [20], в то время как G. King и T. Krzywinska сводят все многообразие компьютерных игр к 4 основным группам: игры-симуляторы, стратегии, экшн-игры и ролевые игры [27]. Некоторые авторы настаивают на выделении в отдельный жанр многопользовательских онлайн игр (ММО), выделяя в качестве ключевых особенностей одновременное присутствие в общем виртуальном мире игры множества игроков, способных взаимодействовать друг с другом [28]. Другие указывают на то, что среди многопользовательских онлайн игр также можно встретить примеры разных традиционных жанров: шутеры, стратегии, РПГ, а также - элементы социальных практик [27]. К недостаткам жанровых классификаций компьютерных игры можно также отнести их внутреннюю противоречивость: например, игра жанра РПГ может содержать задачи разных типов: сложные боевые сцены жанра «экшн», мини-игры «аркады», сложные логические головоломки. R. Clarke с соавторами [20] указывают на то, что некоторые популярные современные игры описываются могут быть отнеси буквально ко всем выделяемым жанрам одновременно. Также, большинство игроков не отдают предпочтения какому-то одному чистому жанру, что усложняет подбор испытуемых для исследований [21].

Как было указано ранее, жанровые классификации компьютерных игр одновременно используют несколько оснований, например - характер игровых действий и интерактивность. Однако для проведения психологических исследований нередко применяются более простые критерии, отвечающие конкретным эмпирическим задачам.

D. Gentile [16] выделяет 5 основных аспектов компьютерной игровой деятельности, потенциально влияющих на игрока: длительность игры; содержание игры (те сценарии, которые в ней реализуются); игровой контекст (то, в какой именно форме подается содержание игры); структура игры (форма подачи игровой информации) и игровая механика (в данном случае речь идет о технической стороне игры - какие навыки и умения в ней задействуются). Исследования игроков в компьютерные игры нередко строятся вокруг только одного из этих аспектов (в то время как жанровые классификации имплицитно включают большинство из них).

Так, сравнение игроков, предпочитающих компьютерные игры, содержащие, либо не содержащие элементы насилия и агрессии апеллирует к содержательным элементам игровой деятельности и отчасти - к игровому контексту. Влияние компьютерных игры с элементами насилия на агрессивные мысли, чувства и действия игроков является предметом научных дискуссий вот уже на протяжении более чем 25 лет. В то время как одни ученые рассматривают связь игрового и реального насилия как эмпирически доказанную (напр., [10]), другие научные группы приводят доказательства обратного $[11,12]$. Деление игр на содержащие и не содержащие агрессивные элементы представляется значительно более простым в практическом применении, чем традиционное жанровое деление, однако имеет существенный недостаток: недоучет эффектов, связанных с игровой структурой и механикой, которые нередко также различаются, например, для игр-«стрелялок» и «мирных» логических головоломок.

В настоящее время все чаще используется деление компьютерных игр на многопользовательские (допускающие совместную игру двух и более людей) и однопользовательские, а также на онлайн и офлайн игры [29]. Это пересекающиеся 
классификации: подавляющее большинство игр в онлайн режиме - многопользовательские, хотя совместная игра в некоторых случаях может быть реализована и в режиме офлайн. В последнем случае количество игроков ограничено, и они взаимодействуют друг с другом в реальности, физически собираясь за одним игровым устройством (компьютером, приставкой). Игра в режиме онлайн (через Интернет), напротив, предполагает возможность взаимодействия с неограниченным количеством игроков, в том числе - из других стран и городов. В типологии D. Gentile эта классификация в большей степени относится к игровому контексту и отчасти - механикам игры.

О.К. Тихомиров и Е.Е. Лысенко [2] определяли специфику ситуации игры один на один с компьютером (однопользовательская игра) следующим образом: компьютер, в отличие от реального игрока, не может в полной мере выполнять функцию противника, поэтому человек скорее играет сам с собой. В такой ситуации игрок может свободно решать творческие и познавательные задачи без оглядки на чужое мнение и оценки. Многопользовательская игра, напротив, задает условия конкуренции, соревнования, а также формирует чувство ответственности перед другими игроками при решении кооперативных задач. Это можно проиллюстрировать исследованием, в котором на материале компьютерной игры Minecraft рассматривалась специфика процесса решения творческих конструкторских задач. Испытуемые, работающие в одиночестве, затрачивали на задачи меньше времени и продуцировали больше вариантов решения по сравнению с теми, кто работал над аналогичными заданиями в диадах [30].

Ученые полагают, что многопользовательские онлайн игры являются более мотивационно привлекательными для геймеров и чаще вызывают аддиктивное поведение $[9,28]$. Характерные для таких игр элементы: непрерывные цепочки заданий, приводящие к появлению эффекта незавершенного действия; случайное подкрепление (в виде распределения редких и ценных игровых предметов между пользователями); поощрение соревновательной мотивации с помощью рейтингов; ежедневные награды и события способствуют увеличению длительности и регулярности игры [31]. Обязательным условием успеха в онлайн играх также является взаимодействие с другими игроками, будь то кооперация или конфронтация: объединение во временные или постоянные группы открывает возможности, недоступные игроку-одиночке $[27,29]$. Однопользовательские игры, в свою очередь, отличаются большей оригинальностью сюжетов, проработанностью игрового мира, разнообразием и новизной игровых механик. В играх этого типа геймер больше контролирует игровые события, также допускаются пользовательские модификации игры [32]. Онлайн и офлайн игры привлекают различных игроков: это демонстрируется, в частности, спецификой игровой мотивации онлайн и офлайн геймеров [29]. При таком делении компьютерных игр, в свою очередь, недостаточно учитывается игровое содержание, а также потенциально связанная с типом игры (в силу специфики мотивации и контекста) продолжительность игровой деятельности. Однако, на наш взгляд, деление компьютерных игр по указанному основанию является достаточно перспективным с точки зрения психологии. Пример собственного исследования, в котором сравниваются когнитивно-стилевые и личностные особенности игроков в компьютерные игры, предпочитающих тот или иной тип игры, приводится в разделе 3 данной статьи.

Наряду с перечисленными выше классификациями по относительно общим, явно заданным в игровом дизайне основаниям, заслуживают внимания и специальные классификации компьютерных игр, разрабатываемые психологами и опирающиеся на психологические основания. В одной из ранних классификаций, А. Г. Шмелев описал семь классов компьютерных игр, выделяемых по задействованным в них психическим функциям [4]:

- «логические игры», стимулирующие формально-логическое мышление;

- «азартные игры» (например, компьютеризированный покер), требующие от игрока интуитивного, иррационального мышления;

- «спортивные игрыl», апеллирующие к ловкости и проворству, сенсомоторной координации, концентрации внимания;

- «военные игры» и «игры-единоборства», как правило, содержащие картины разрушений и элементы жестокости или насилия и способствующие развитию эмоциональной устойчивости к неудачам, настойчивости. Такие игры могут служить в

International Culture \& Technology Studies, Vol. 3, No. 4 
качестве социально приемлемого инструмента разрядки агрессивных импульсов, но противопоказаны людям с неустойчивой психикой;

- игры типа «преследование-избегание», для которых характерно включение в игровой процесс интуитивного компонента мышления и эмоционально-чувственного восприятия;

- «авантюрные игры» (игры-приключения, или «аркады»), апеллирующие к нагляднодейственному мышлению, либо активно задействующие оперативную память для абстрактного моделирования недостающих элементов зрительного поля;

- «игры-тренажеры», обучающие игры, отвечающие требованиям конкретной профессиональной деятельности.

Классификация А.Г. Шмелева, таким образом, отталкивается от тех качеств, развитие которых может быть достигнуто с помощью игры того или иного типа, однако подчеркивается, что этого может и не произойти при обычной регулярной игре для развлечения.

Другая психологическая классификация компьютерных игр была предложена Р.Е. Радевой и Е.О. Смирновой [33] и является вариантом жанрового деления компьютерных игр с добавлением в качестве основного критерия ролевой позиция игрока: «над игровой ситуацией», «вне ее» или «внутри нее», а также ведущей мотивации игры. Позиция игрока определяет возможность принятия той или иной роли, что особенно важно в контексте изучения игр детей, в частности - дошкольников, для которых сюжетно-ролевая игра является ведущей деятельностью. Таким образом, авторами выделяются следующие категории игр (с указанием оснований категоризации):

- «головоломки» и компьютеризованные традиционные игры; являются вариантом «игр с правилами», но не дают игроку принимать какую-либо ролевую позицию в них;

- «аркады»» (платформеры) - подразумевают позицию «вне игровой ситуации», однако также не задействуют ролевое поведение, так как персонаж игры является лишь формальным элементом и не несет смысловой нагрузки для играющего; ведущий мотив - достижение успеха;

- «стратегии» - ролевая позиция игрока сводится к формальному обозначению полномочий (возможностей); ведущие мотивы - достижение успеха, власть, познавательный мотив;

- «симуляторы» (в эту категорию также попадает большинство игр жанра «экшн») ролевая позиция «внутри ситуации», задающаяся, как правило, видом «от первого лица»;

- «игры-повествования» (состоит из двух классов игр - «квестов» и РПГ) - ролевая позиция игрока «вне игровой ситуации»; мотивация может быть связана с реализацией неосуществимых в реальности желаний и импульсов.

В то время как классификация А.Г. Шмелева в большей степени подходит для анализа компьютерной игровой деятельности взрослых игроков со сформированной мотивационносмысловой сферой личности, классификации Р.Е. Радевой и Е.О. Смирновой [33] позиционируется как средство для анализа компьютерной игровой деятельности детей, в том числе - дошкольного возраста, что приобретает особую актуальность в настоящее время, в связи с стремительным распространением компьютерных игр среди детей младше 5 лет. В то же время, наряду с ориентацией на психические процессы и их преобразование в игровой деятельности, психологические классификации компьютерных игр остаются сравнительно малоизвестными. Их практическое применение в психологических исследованиях также осложняется необходимостью проведения экспертного анализа каждый раз, когда необходимо отнести ту или иную игру к определенной категории. Тем не менее, классификации компьютерных игр по психологическим основаниям могут быть особенно полезны как для разработки и изучения так называемых «серьезных» компьютерных игр, ставящих перед собой задачу обучения, развития, просвещения игроков, а не развлечение. 
Еще одним актуальным и специфическим направлением, в рамках которого в ближайшее время может потребоваться разработка специальных, в том числе - ориентированных на психологические аспекты - классификаций, является компьютерный или киберспорт. В рамках соревновательной компьютерной игры представлены различные жанры, такие как «шутеры», «стратегии», многопользовательские онлайновые РПГ-игры, однако мотивация профессиональных игроков существенно отличается от мотивации игры в целях развлечения [34] - таким образом, можно говорить о том, что потенциальное воздействие занятий профессиональным компьютерным спортом может отличаться от известных психологических последствий тех же самых игры на обычных пользователей.

\section{3. Исследование когнитивно-стилевой и личностной специфики принятия решений у геймеров, предпочитающих онлайн и офлайн игры}

\section{1. Постановка проблемы и дизайн исследования}

Предметом изучения являются когнитивно-стилевые и личностные особенности геймеров, предпочитающих игры разных типов. Когнитивные стили как индивидуальные устойчивые способы восприятия и первичной переработки информации влияют на протекание всех видов деятельности человека. В исследовании рассмотрены следующие когнитивные стили:

- полезависимость-поленезависимость (ПЗ-ПНЗ) характеризует способность человека выделять в зрительном поле значимые элементы (ПНЗ-стиль), либо стремление воспринимать его целостно (ПЗ-стиль) [35]. ПЗ-ПНЗ стиль также связан с механизмами непроизвольного интеллектуального контроля, вниманием и кратковременной памятью, характеристиками, которые традиционно изучаются в контексте компьютерных игр;

- импульсивность-рефлективность описывает специфику решения задач - склонность давать быстрые, но неточные (импульсивность), либо медленные и точные ответы (рефлективность). Импульсивность часто рассматривается как характерная особенность геймеров, но эмпирические данные об этом противоречивы $[6,7]$.

Были сформулированы следующие гипотезы исследования:

- геймеры, предпочитающие компьютерные игры разных типов, различаются по степени выраженности у них когнитивного стиля полезависимость-поленезависимость;

- геймеры, предпочитающие компьютерных игры разных типов, различаются по степени уровня импульсивности и склонности к риску; они более импульсивны, чем люди, не играющие в компьютерные игры.

Выборка исследования: 223 испытуемых в возрасте 18-35 лет (M = 23,7; $\mathrm{SD}=4,6$ лет), из них 91 мужчина и 132 женщины. 150 человек (65 мужчин и 85 женщин) выполнили все методики исследования, а 73 (26 мужчин и 47 женщин) ответили на вопросы интервью и заполнили личностный опросник.

Игровые предпочтения испытуемых выявлялись в ходе специально разработанного структурированного интервью, включающего вопросы демографического характера и непосредственно связанные с компьютерными играми. Было установлено, что больше половины $(60 \%)$ испытуемых не могут выделить предпочитаемый жанр игры. При разделении игр на два типа: онлайн (многопользовательские) или офлайн (однопользовательские) игры, большинство испытуемых смогли четко обозначить свои предпочтения. А именно: 50\% испытуемых играют либо в онлайн, либо в офлайн игры; $45 \%$ имели опыт игры в компьютерные игры обоих типов, но смогли однозначно указать более интересный и привлекательный для них тип; $5 \%$ затруднялись с ответом, но смогли сформулировать свои предпочтения в ходе интервью. В целом, геймеры, предпочитающие онлайн игры, отмечали значимость социальной составляющей игры, внутриигровое общение, реже - возможность ощутить свое превосходство над другими игроками. Геймеры, предпочитающие офлайн игры, указывали, что в игре их привлекает сюжет, проработанность игрового мира, возможность погрузиться в другую реальность, сложные игровые

International Culture \& Technology Studies, Vol. 3, No. 4 
задачи. Предложенные игроками мотивировки, таким образом, относятся к разным факторам игровой мотивации по $\mathrm{N}$. Yеe [15], и онлайн геймеры демонстрируют более выраженную социальную мотивацию, что соответствует результатам Т. Наiney с соавторами [29]. Разделение игроков по предпочтению онлайн и офлайн игр представляется обоснованным также с точки зрения специфики дизайна этих игр.

Описание выборки исследования представлено в таблице 1. В контрольную группу вошли испытуемые, не играющие в компьютерные игры и не интересующиеся ими.

Таблица 1. Характеристики выборки

\begin{tabular}{|c|c|c|}
\hline \multicolumn{2}{|c|}{$\begin{array}{c}\text { Исследуемая группа: } \\
\text { всего } 133 \text { испытуемых, } 63 \text { мужчины, } 70 \text { женщин, } \\
\text { средний возраст 23,6 } 44,5 \text { лет }\end{array}$} & \multirow{2}{*}{$\begin{array}{c}\text { Контрольная группа: } \\
90 \text { испытуемых, } \\
28 \text { муж., } 62 \text { жен., } \\
\text { ср. возраст: } 23,7 \pm 4,9 \text { лет }\end{array}$} \\
\hline $\begin{array}{c}\text { Играют в онлайн игры: } \\
69 \text { испытуемых: } 41 \text { муж., } 28 \text { жен., } \\
\text { ср. возраст } 23,9 \pm 5 \text { лет }\end{array}$ & $\begin{array}{c}\text { Играют в офлайн игры: } \\
64 \text { испытуемый: } 22 \text { муж., } 42 \text { жен. } \\
\text { ср. возраст } 23,3 \pm 3,9 \text { лет }\end{array}$ & \\
\hline
\end{tabular}

Методики исследования:

- методика «Сравнение похожих рисунков» Дж. Кагана - для измерения когнитивного стиля импульсивность-рефлективность;

- методика «Включенные фигуры» Г. Уиткина - для измерения когнитивного стиля полезависимость-поленезависимость;

- опросник «Импульсивность 7» (И7) Г. Айзенка и С. Айзенк в адаптации Т.В. Корниловой и А.А. Долныковой: шкалы импульсивности, склонности к риску как поиску острых ощущений, эмпатии.

\section{2. Результаты и обсуждение}

\section{Когнитивный стиль полезависимость-поленезависимость:}

В методике «Включенные фигуры» Г. Уиткина в качестве показателя полезависимогополенезависимого когнитивного стиля выступает средняя скорость обнаружения простой фигуры в сложной (в секундах; чем меньше времени требуется на решение заданий, тем выше поленезависимость). Однофакторный дисперсионный анализ показал значимые различия между геймерами, предпочитающими разные типы игр $(\mathrm{F}=7,099 ; \mathrm{p}=0,001)$. Попарное межгрупповое post hoc сравнение по методу Бонферрони выявило статистически значимые различия только между группами офлайн игроков и контрольной группой $(p=0,001)$. Различия по данному критерию между онлайн и офлайн игроками $(\mathrm{p}=0,385)$, а также онлайн игроками и контрольной группой $(\mathrm{p}=0,085)$ не значимы. Среднее время обнаружения простой фигуры в сложной в секундах составило $24,4 \pm 10,9$ с в контрольной группе; $20,2 \pm 9,6$ с в группе онлайн-геймеров; $16,9 \pm 8,3$ с в группе офлайн-геймеров.

Таким образом, игроки, предпочитающие играть в офлайн компьютерные игры, более поленезависимы по сравнению с другими группами.

\section{Когнитивный стиль импульсивность-рефлективность:}

В методике «Сравнение похожих рисунков» Дж. Кагана измеряются два показателя, определяющие когнитивный стиль импульсивность-рефлективность: количество ошибок, совершаемых испытуемым (показатель точности ответов) и среднее время первого ответа (в секундах; показатель когнитивного темпа). Импульсивный когнитивный стиль характеризуется большим количеством ошибок и быстрыми ответами, рефлективный - малым количеством ошибок и медленными ответами. 
Между группами не было выявлено различий по показателю среднего времени первого ответа (согласно непараметрическому критерию Краскала-Уоллеса, $\mathrm{X}^{2}=1,988 ; \mathrm{p}=0,370$ ). В то же время испытуемые значимо различались по количеству ошибок: $\mathrm{X}^{2}=16,405 ; \mathrm{p}=0,000$. Среднее количество ошибок в группе онлайн-геймеров: $6,7 \pm 4,6$; в группе офлайн-геймеров: $3,6 \pm 4,4$, в контрольной группе: 7,5 \pm 6.

В таблице 2 представлены результаты попарного сравнения групп испытуемых, предпочитающих разные типы компьютерных игр с помощью непараметрического критерия Манна-Уитни.

Таблица 2. Сравнение групп геймеров, предпочитающих онлайн и офлайн игры по количеству ошибок и среднему латентному времени первого ответа в тесте Дж. Кагана

\begin{tabular}{|c|c|c|c|c|c|c|}
\hline \multirow{2}{*}{} & \multicolumn{2}{|c|}{ Контрольная группа } & \multicolumn{2}{c|}{ Офлайн игроки } & \multicolumn{2}{c|}{ Онлайн игроки } \\
\cline { 2 - 7 } & $\begin{array}{c}\text { Кол-во } \\
\text { ошибок }\end{array}$ & $\begin{array}{c}\text { Ср. время 1- } \\
\text { ого ответа }\end{array}$ & $\begin{array}{c}\text { Кол-во } \\
\text { ошибок }\end{array}$ & $\begin{array}{c}\text { Ср. время 1- } \\
\text { ого ответа }\end{array}$ & $\begin{array}{c}\text { Кол-во } \\
\text { ошибок }\end{array}$ & $\begin{array}{c}\text { Ср. время 1- } \\
\text { ого ответа }\end{array}$ \\
\hline $\begin{array}{c}\text { Контрольная } \\
\text { группа }\end{array}$ & - & - & $\begin{array}{c}\mathbf{U = 7 3 6 , 5} \\
\mathbf{p}=\mathbf{0 , 0 0 1}\end{array}$ & $\begin{array}{c}\mathrm{U}=1053,5 \\
\mathrm{p}=0,222\end{array}$ & $\begin{array}{c}\mathrm{U}=1418,5 \\
\mathrm{p}=0,753\end{array}$ & $\begin{array}{c}\mathrm{U}=1279,0 \\
\mathrm{p}=0,245\end{array}$ \\
\hline Офлайн игроки & $\begin{array}{c}\mathbf{U}=\mathbf{7 3 6 , 5} \\
\mathbf{p}=\mathbf{0 , 0 0 1}\end{array}$ & $\begin{array}{c}\mathrm{U}=1053,5 \\
\mathrm{p}=0,222\end{array}$ & - & - & $\begin{array}{c}\mathbf{U}=\mathbf{5 4 2 , 0} \\
\mathbf{p}=\mathbf{0 , 0 0 0}\end{array}$ & $\begin{array}{c}\mathrm{U}=993,0 \\
\mathrm{p}=0,926\end{array}$ \\
\hline Онлайн игроки & $\begin{array}{c}\mathrm{U}=1418,5 \\
\mathrm{p}=0,753\end{array}$ & $\begin{array}{c}\mathrm{U}=1279,0 \\
\mathrm{p}=0,245\end{array}$ & $\begin{array}{c}\mathbf{U = 5 4 2 , 0} \\
\mathbf{p}=\mathbf{0 , 0 0 0}\end{array}$ & $\begin{array}{c}\mathrm{U}=993,0 \\
\mathrm{p}=0,926\end{array}$ & - & - \\
\hline
\end{tabular}

Группа офлайн игроков статистически значимо отличалась как от контрольной группы, так и от группы геймеров по количеству ошибок в тесте Дж. Кагана. Таким образом, офлайн игроки наиболее точно справляются с методикой и более рефлективны.

\section{Импульсивность, склонность к риску, эмпатия:}

Опросник И7 измеряет следующие личностные особенности: импульсивность как склонность вести себя необдуманно, не осознавая последствий своих действий; склонность к риску как осознанное стремление к рискованным действиям, в том числе - экстремальным формам активности; эмпатию как способность к сопереживанию, заражению эмоциями, вхождению в эмоциональное состояние другого человека.

Согласно непараметрическому критерию Краскала-Уоллеса, испытуемые, предпочитающие игры разного типа, значимо не отличались по уровню импульсивности $\left(\mathrm{X}^{2}=0,011 ; \mathrm{p}=0,995\right)$, однако различались по склонности к риску $\left(\mathrm{X}^{2}=15,987 ; \mathrm{p}=0,000\right)$ и эмпатии $\left(\mathrm{X}^{2}=14,196 ; \mathrm{p}=0,001\right)$. Средние баллы по этим шкалам представлены в Таблице 3.

Таблица 3. Средние баллы испытуемых, предпочитающих онлайн и офлайн игры по шкалам Импульсивности, Склонности к риску и Эмпатии опросника И7

\begin{tabular}{|c|c|c|c|}
\hline & Импульсивность & Склонность к риску & Эмпатия \\
\hline Онлайн-игроки & $1,1 \pm 5,2$ & $3 \pm 3,7$ & $3,5 \pm 3,9$ \\
\hline Офлайн-игроки & $0,9 \pm 5,4$ & $0,4 \pm 4,2$ & $4,1 \pm 3,3$ \\
\hline Не играют & $1 \pm 5,4$ & $0,7 \pm 4,1$ & $5,5 \pm 3,2$ \\
\hline
\end{tabular}

Попарное сравнение групп с помощью непараметрического критерия Манна-Уитни показало, что склонность к риску у онлайн игроков значимо выше, чем у офлайн игроков $(\mathrm{U}=1435,5 ; \mathrm{p}=0,000)$ и у контрольной группы $(\mathrm{U}=2125,0 ; \mathrm{p}=0,001)$, а показатели офлайн и контрольной групп значимо не различались $(U=2776,5 ; p=0,701)$. По уровню эмпатии не выявлены значимые различия между онлайн и офлайн игроками $(U=2136,5 ; p=0,744)$, но обе группы 
геймеров имели более низкие показатели по сравнению с контрольной группой $(U=2153,0 ; p=0,001$ и $\mathrm{U}=2089,0 ; \mathrm{p}=0,003$ для онлайн и офлайн игроков соответственно).

Таким образом, среди игроков в компьютерные игры высокие показатели склонности к риску как поиску сильных, экстремальных ощущений наиболее присущи онлайн геймерам. Также, все геймеры, независимо от предпочитаемого типа игр, характеризуются более низкой эмпатией по сравнению с не играющими в компьютерные игры испытуемыми.

Проведенное исследование выявило когнитивно-стилевую и личностную специфику, характерную для онлайн и офлайн геймеров. Когнитивно-стилевые особенности (высокая поленезависимость, низкая импульсивность) более выражены у офлайн игроков, в то время как личностные особенности (высокая склонность к риску, низкая эмпатия) более выражены в группе онлайн игроков. Можно предположить, что показанные различия связаны со спецификой компьютерной игровой деятельности, а также с особенностями мотивации компьютерной игры. Так, офлайн компьютерные игры, характеризующиеся детальной проработанностью игрового мира, сюжета, зачастую - сложными логическими головоломками, в большей степени обращаются к когнитивным функциям игрока: логическому и пространственному мышлению, зрительному вниманию. При этом на принятие решений предоставляется больше времени по сравнению с онлайн играми, требующими высокой скорости реакции. Онлайн компьютерные игры более динамичны, активны, предъявляют иные требования к когнитивным способностям игроков, сопряжены с большим риском, так как в них отсутствует возможность в точности переиграть какое-либо событие, результаты ошибки могут отразиться на игровой статистике или авторитете игрока. В силу этого онлайн игры, вероятно, скорее привлекают игроков, склонных к поиску острых ощущений, в то время как более осторожные игроки предпочитают игру один на один с компьютером с максимально четко заданным сценарием, в ситуации «поправимой ошибки» [4]. Что касается сниженной эмпатии у геймеров, характер исследования не позволяет установить причинно-следственные связи, однако можно предполагать, что увлеченность компьютерными играми не способствует (и возможно - вредит) развитию сопереживания. Возможно также, что для людей $\mathrm{c}$ изначально сниженной эмпатией компьютерные игры являются более привлекательным способом времяпрепровождения в силу упрощенного и относительно безопасного (в сравнении с реальным общением) характера коммуникации.

Предложенное разделение компьютерных игр на онлайн и офлайн игры показало себя оправданным и позволило выявить различия в психологических особенностях геймеров, что согласуется с более ранними исследованиями.

\section{4. Выводы и заключение}

По результатам проделанной работы можно сделать следующие выводы:

- компьютерные игры характеризуются качественным многообразием, которое необходимо учитывать при проведении эмпирических исследований. Для этого целесообразно использовать классификации компьютерных игр по различным основаниям, в т.ч. - бинарным, например, онлайн и офлайн игры;

- наиболее выраженной спецификой когнитивно-стилевой сферы (высокой поленезависимостью и рефлективностью) характеризуются игроки, предпочитающие офлайн компьютерные игры;

- геймеры не отличаются от не играющих в компьютерные игры испытуемых по уровню когнитивной и личностной импульсивности, однако для игроков в онлайн игры характерна высокая склонность к риску как к поиску острых ощущений;

- низкие показатели эмпатии характерны для геймеров с любыми игровыми предпочтениями по сравнению с неиграющими испытуемыми.

Исследования компьютерных игр и игроков («геймеров») ведутся на протяжении уже нескольких десятков лет. Несмотря на множество книг и статей, посвященных данной проблематике, в психологии до сих пор отсутствуют единые взгляды относительно того, может ли увлечение компьютерными играми привести к каким-либо существенным последствиям для психики игроков, а актуальность подобных исследований лишь возрастает по мере того, как

Культура и технологии, Том 3, № 4 
компьютерные превращаются не только в глобальную индустрию развлечений, но также - в зрелищные спортивные соревнования, средство обучения, социализации и т.д. Анализ возможных классификаций компьютерных игр и оснований для новых классификаций, приведенный в этой статье служит, на наш взгляд, иллюстрацией того, насколько многообразны компьютерные игры - в том числе, по своим психологическим аспектам и характеристикам, а проведенное эмпирическое исследование продемонстрировало возможность применения одной из классификаций для более точной интерпретации результатов. Напротив, недостаточно внимательное отношение к различным аспектам игровой деятельности, мотивации игроков, структурным элементам игры, может служить источником как недооценки, так и переоценки тех или иных потенциальных игровых эффектов, что необходимо учитывать как при проведении собственных исследований, так и при анализе уже имеющихся работ.

Работа выполнена при поддержке РНФ, проект № 18-18-00365.

\section{Литература}

[1] Turkle Sh. The Second Self: Computers and the Human Spirit. New York: Simon \& Schuster, 1984. 362 p.

[2] Тихомиров О.К., Лысенко Е.Е. Психология компьютерной игры // Новые методы и средства обучения. 1988. Вып. 1. М.: Знание. С. 30-66.

[3] Subrahmanyam K., Greenfield P.M. Effect of video game practice on spatial skills in girls and boys // Journal of Applied Developmental Psychology. 1994. Vol. 15. P. 13-32.

[4] Шмелев А.Г. Мир поправимых ошибок // Вычислительная техника и ее применение. Компьютерные игры. 1988. № 3. С. 16-84.

[5] Игра со всех сторон: Современные исследования, междисциплинарный подход, практические рекомендации, взгляд в будущее / Под ред. Е.С. Жорняк. М.: Прагматика культуры, 2003. 432 с.

[6] Video game playing, attention problems, and impulsiveness: evidence of bidirectional causality / D.A. Gentile [et al.]. // Psychology of popular media culture. 2012. № 1. P. 62-70.

[7] Metcalf O., Pammer K. Impulsivity and related neuropsychological features in regular and addictive first person shooter gaming // Cyberpsychology, behavior and social networking. 2014. Vol. 17. № 3. P. 147-152.

[8] Богачева Н.В., Войскунский А.Е. Когнитивные стили и импульсивность у геймеров с разным уровнем игровой активности и предпочитаемым типом игр // Психология. Журнал Высшей Школы экономики. 2015. T. 12, № 1. C. 29-53.

[9] Collins E., Freeman J., Chamarro-Premuzic T. Personality traits associated with problematic and nonproblematic massively multiplayer online role playing game use // Personality and individual differences. 2012. № 52. P. 133-138.

[10] Violent video game effects on aggression, empathy, and prosocial behavior in eastern and western countries: a meta-analytic review / C.A. Anderson [et al.]. // Psychological Bulletin. 2010. Vol. 136. № 2. P. 151-173.

[11] Olson C., Kutner L. Viewpoints and flashpoints in the study of video game violence and aggression // Psychology. Journal of Higher School of Economics. 2015. Vol. 12, № 1. P. 13-28.

[12] Markey P.M., Ferguson C.J. Moral Combat: Why the War on Violent Video Games Is Wrong. Dallas: BenBella Books, 2017. $256 \mathrm{p}$.

[13]Playing Super Mario induces structural brain plasticity: gray matter changes resulting from training with a commercial video game / S. Kühn [et al.]. // Molecular Psychiatry. 2014. Vol. 19, № 2. P. 265-271.

[14] Impact of video games on plasticity of the hippocampus / G.L. West [et al.]. // Molecular Psychiatry. 2018. Vol. 23. P. 1566-1574.

[15] Yee N. Motivations of Play in Online Games // CyberPsychology and Behavior. 2006. Vol. 9. P. 772-775.

[16] Gentile D. The Multiple Dimensions of Video Game Effects // Child Development Perspectives. 2011. Vol. 5. P. 75-81.

[17]List of Every Video Game Ever [Электронный ресурc]. 2014. URL: https://pastebin.com/EuxZMbWT (дата обращения: 25.12.2018)

[18] Alternate List of Every Video Game Ever [Электронный pecypc]. 2014. URL: https://pastebin.com/U4n85p8s (дата обращения 25.12.2018)

[19]Ruggiero L. The Number of Steam Games Out There Has Reached 10,000 Mark [Электронный ресурс] // SegmentNext. 2016. URL: https://The Number of Steam Games Out There Has Reached 10,000 Mark.com/2016/10/13/number-of-steam-games-10k/ (дата обращения: 25.12.2018)

[20] Clarke R.I., Lee J.H., Clark N. Why Video Game Genres Fail: A Classificatory Analysis // Games and Culture. 2015. Vol. 12, № 5. P. 445-465.

[21] Аветисова А.А. Психологические особенности игроков в компьютерные игры // Психология. Журнал Высшей Школы Экономики. 2011. Т. 8. № 4. С. 35-58.

International Culture \& Technology Studies, Vol. 3, No. 4 
[22] Clearwater D.A. What Defines Video Game Genre? Thinking about Genre Study after the Great Divide // Loading... The Journal of the Canadian Game Studies Association. 2011. Vol. 5. № 8. P. 29-49

[23] Green S., Bavelier D. Action computer game modifies visual selective attention // Nature. 2003. Vol. 423. P. 523-537.

[24] Green S., Bavelier D. Action-video-game experience alters the spatial resolution of vision // Psychological science. 2007. Vol. 18, № 1. P. 88-94.

[25] Glass B.D., Maddox W.T., Love B.C. Real-Time Strategy Game Training: Emergence of a Cognitive Flexibility Trait [Электронный pecypc] // Plos One. 2013. Vol. 8, № 8. URL: http://journals.plos.org/plosone/article/file?id=10.1371/journal.pone.0070350\&type=printable (дата обращения 25.12.2018)

[26] Kühn S., Gallinat J. Amount of lifetime video gaming is positively associated with entorhinal, hippocampal and occipital volume // Molecular Psychiatry. 2014. Vol. 19, № 7. P. 842-847.

[27] Apperley T. Genre and game studies: Towards a critical approach to video game genres // Simulation \& Gaming. 2006. Vol. 37. № 1. P. 6-23.

[28] Sellers M. Designing the experience of interactive play // Playing video games. Motives, Responses and Consequences / P.Vorderer, J.Bryant (eds.). Mahwah, New Jersey: Lawrence Erbaum. 2006. P. 9-22.

[29] The differences in motivations of online game players and offline game players: a combined analysis of three studies at higher education level / T. Hainey [et al.]. // Computers \& Education. 2011. Vol. 57. № 4. P. 2197 2211.

[30] Creativity in online gaming: individual and dyadic performance in Minecraft / A.E. Voiskounsky [et al.] // Psychology in Russia: State of the Art. 2017. Vol. 10. №. 4. P. 144-161.

[31] Griffiths M. Online computer gaming: Advice for parents and teachers // Education and Health. 2009. Vol. 27. № 1. P. 3-6.

[32] Kow Y.M., Nardi B. Culture and Creativity: World of Warcraft Modding in China and the US // Online Worlds: Convergence of the Real and the Virtual / Bainbridge W. (eds). Human-Computer Interaction Series. Berlin: Springer, 2009. P. 21-41.

[33] Смирнова Е.О., Радева Р.Е. Психологические особенности компьютерных игр: новый контекст детской субкультуры // Образование и информационная культура. Социологические аспекты. М.: Центр социологии образования РАО, 2000. С. 330-336.

[34] The Psychology of Esports: A Systematic Literature Review / F. Bányai [et al.] // Journal of Gambling Studies. 2018. doi:10.1007/s10899-018-9763-1

[35] Холодная М.А. Когнитивные стили: О природе индивидуального ума. СПб: Питер, 2004. 384 с.

\title{
The Problem of Videogames Classification in the Context of Computer Gamers Psychological Research
}

\author{
N. Bogacheva ${ }^{1}$, A. Voiskounsky ${ }^{2}$ \\ ${ }^{1}$ FGAOU VO I.M. Sechenov First Moscow State Medical University (Sechenov University), Russia \\ ${ }^{2}$ FGBOU VO M.V. Lomonosov Moscow State University, Russia
}

\begin{abstract}
The article describes different approaches to the classification of video games in psychology and other sciences. Some of the video games classifications are often introduced in psychological research, but they have their own potential advantages and disadvantages. One of the most popular video games classifications is the "genre classification", which is also often criticized by computer scientists for its controversies. There are, in fact, several different variants of genre classifications of video games, which sometimes make research analysis even more difficult. Along with the genre classification of video games, the article describes different research based on simpler classification criteria, such as game content elements (e.g. violence), number of players, game type (online or offline). Among them, there are specific video games classifications, built on psychological grounds and for the purpose of psychological research. These classifications describe different video game motives, skills acquired in the game, gamers role position., etc. The theoretical review demonstrates the heterogeneity of computer gamers` psychological characteristics depending on their gaming preferences. The empirical part of the study describes cognitive-style and personality (empathy, impulsivity, risk appetite) research of computer gamers, who prefer online and offline games (total $\mathrm{n}=223$; age $18-35, \mathrm{M}=23.7 ; \mathrm{SD}=4.6$ years). Among three groups (online gamers, $\mathrm{n}=$ 69; offline gamers, $n=64$; non-gamers, $n=90$ ), offline gamers were found to be significantly more field-independent (cognitive style, measure by H. Witkin`s EFT) then the non-gamers group ( $\mathrm{p}=$ 0.001) and also less impulsive (cognitive style, measured by J. Kagan`s MFFT) then both online
\end{abstract}


gamers $(\mathrm{p}=0.000)$ and non-gamers $(\mathrm{p}=0.001)$. Online gamers, though, showed the most distinct personality specifics among the three groups (according to Eysencks` I7 Scale). They were found to be more venturesome then both other groups $(\mathrm{p}=0.001)$. Both gamers group were also found to be less empathetic than the control group, according to the same test.

Keywords: videogame; computer gamers; videogames classification; online games; offline games; impulsiveness; cognitive styles; field-dependence; e-sports

\section{References}

[1] Turkle, Sh. (1984) The Second Self: Computers and the Human Spirit. New York: Simon \& Schuster. 362 p.

[2] Tikhomirov, O.K., Lysenko, E.E. (1988) Psihologiya kompyuternoj igry [The Psychology of Computer Game]. Novye metody i sredstva obucheniya [New methods and tools of education]. Issue 1. Moscow. Znanie Publ. pp. 30-66.

[3] Subrahmanyam, K., Greenfield, P.M. (1994) Effect of video game practice on spatial skills in girls and boys. Journal of Applied Developmental Psychology. Vol. 15. 13-32. https://doi.org/10.1016/0193-3973(94)90004-3

[4] Shmelev, A.G. (1988) Mir popravimyh oshibok [The World of Reparable Mistakes]. Vychislitelnaya tekhnika $i$ ee primenenie. Kompyuternye igry. [Computer Technology and Its Application. Computer Games]. No. 3. 1684.

[5] Fromberg, D., Bergen, D., eds. (1998) Play from birth to twelve and beyond: Contexts, perspectives, and meanings. New York, London. Garland Publ. 578 p.

[6] Gentile, D.A., Swing, E.L, Choon, G.L., Khoo, A. (2012) Video game playing, attention problems, and impulsiveness: evidence of bidirectional causality. Psychology of popular media culture. No. 1. 62-70. http://dx.doi.org/10.1037/a0026969

[7] Metcalf, O., Pammer, K. (2014) Impulsivity and related neuropsychological features in regular and addictive first person shooter gaming. Cyberpsychology, behavior and social networking. Vol. 17. No. 3. 147-152. doi: 10.1089/cyber.2013.0024

[8] Bogacheva, N.V., Voiskounsky, A.E. (2015) Kognitivnye stili i impulsivnost u gejmerov s raznym urovnem igrovoj aktivnosti i predpochitaemym tipom igr [Cognitive Styles and Impulsivity of Videogamers with Different Levels of Gaming Activity and Preference of Different Game Types]. Psihologiya. Zhurnal Vysshej Shkoly Ekonomiki. [Psychology. Journal of the Higher School of Economics]. Vol. 12. No. 1. 29-53.

[9] Collins, E., Freeman, J., Chamarro-Premuzic, T. (2012) Personality traits associated with problematic and nonproblematic massively multiplayer online role playing game use. Personality and individual differences. Vol. 52. No. 2. 133-138. https://doi.org/10.1016/j.paid.2011.09.015

[10] Anderson, C.A., Ihori, N., Bushman, B.J., Rothstein, H.R., Shibuya, A., Swing, E.L., Sakamoto, A., Saleem, M. (2010) Violent video game effects on aggression, empathy, and prosocial behavior in eastern and western countries: a meta-analytic review. Psychological Bulletin. Vol. 136. No. 2. 151-173. doi: 10.1037/a0018251

[11] Olson, C., Kutner, L. (2015) Viewpoints and flashpoints in the study of video game violence and aggression. Psychology. Journal of Higher School of Economics. Vol. 12. No. 1. 13-28.

[12] Markey, P.M., Ferguson, C.J. (2017) Moral Combat: Why the War on Violent Video Games Is Wrong. Dallas: BenBella Books. 256 p.

[13] Kühn, S., Gleich, T., Lorenz, R.C., Lindenberger, U., Gallinat, J. (2014) Playing Super Mario induces structural brain plasticity: gray matter changes resulting from training with a commercial video game. Molecular Psychiatry. Vol. 19. No. 2. 265-271. doi: 10.1038/mp.2013.120

[14] West, G.L., Konishi, K., Diarra, M., Benady-Chorney, J., Drisdelle, B.L., Dahmani, L., Sodums, D.J., Lepore, F., Jolicoeur, P., \& Bohbot, V.D. (2018). Impact of video games on plasticity of the hippocampus. Molecular Psychiatry. Vol. 23. 1566-1574. doi: 10.1038/mp.2017.155

[15] Yee, N. (2006) Motivations of Play in Online Games. CyberPsychology and Behavior. Vol. 9. 772-775. doi: 10.1089/cpb.2006.9.772

[16] Gentile, D. (2011). The Multiple Dimensions of Video Game Effects. Child Development Perspectives. Vol. 5. 75-81. 10.1111/j.1750-8606.2011.00159.x.

[17] List of Every Video Game Ever (2014). Available at: https://pastebin.com/EuxZMbWT (accessed date: $12 / 25 / 2018)$

[18] Alternate List of Every Video Game Ever (2014) Available at: https://pastebin.com/U4n85p8s (accessed date: $12 / 25 / 2018)$

[19] Ruggiero, L. (2016) The Number of Steam Games Out There Has Reached 10,000 Mark. SegmentNext. Available at: https://The Number of Steam Games Out There Has Reached 10,000 Mark.com/2016/10/13/number-of-steam-games-10k/ (accessed date: 12/25/2018)

[20] Clarke, R.I., Lee, J.H., Clark, N. (2015). Why Video Game Genres Fail: A Classificatory Analysis. Games and Culture. Vol. 12, No 5. 445-465. doi:10.1177/1555412015591900

International Culture \& Technology Studies, Vol. 3, No. 4 
[21] Avetisova, A.A. (2011) Psihologicheskie osobennosti igrokov v kompyuternye igry [Psychological Features of Computer Gamers]. Psihologiya. Zhurnal Vysshej Shkoly Ekonomiki. [Psychology. Journal of the Higher School of Economics]. Vol. 8. No 4. 35-58.

[22] Clearwater, D.A. (2011) What Defines Video Game Genre? Thinking about Genre Study after the Great Divide. Loading... The Journal of the Canadian Game Studies Association. Vol. 5. No. 8. 29-49.

[23] Green, S., Bavelier, D. (2003) Action computer game modifies visual selective attention. Nature. Vol. 423. 523-537. doi: 10.1038/nature01647

[24] Green, S., Bavelier, D. (2007) Action-video-game experience alters the spatial resolution of vision. Psychological science. Vol. 18. No 1. 88-94.

[25] Glass, B.D., Maddox, W.T., Love, B.C. (2013) Real-Time Strategy Game Training: Emergence of a Cognitive Flexibility Trait. Plos One. Vol. 8, No 8. Available at: http://journals.plos.org/plosone/article/file?id=10.1371/journal.pone.0070350\&type=printable (accessed date 12/25/2018). doi: 10.1371/journal.pone.0070350

[26]Kühn, S., Gallinat. J. (2014) Amount of lifetime video gaming is positively associated with entorhinal, hippocampal and occipital volume. Molecular Psychiatry. Vol. 19. No. 7. 842-847. doi: 10.1038/mp.2013.100

[27] Apperley, T. (2006) Genre and game studies: Towards a critical approach to video game genres. Simulation \& Gaming. Vol. 37. No. 1. 6-23. https://doi.org/10.1177/1046878105282278

[28] Sellers, M. (2006) Designing the experience of interactive play. Playing video games. Motives, Responses and Consequences / P.Vorderer, J.Bryant (eds.). Mahwah, New Jersey: Lawrence Erbaum. 2006. pp. 9-22.

[29] Hainey, T., Connolly, T., Stansfield, M., Boyle, E. (2011) The differences in motivations of online game players and offline game players: a combined analysis of three studies at higher education level. Computers \& Education. Vol. 57. No. 4. 2197-2211. https://doi.org/10.1016/j.compedu.2011.06.001

[30] Voiskounsky, A.E., Yermolova, T.D., Yagolkovskiy, S.R., Khromova, V.M. (2017) Creativity in online gaming: individual and dyadic performance in Minecraft. Psychology in Russia: State of the Art. Vol. 10. No. 4. 144-161.

[31] Griffiths, M. (2009) Online computer gaming: Advice for parents and teachers. Education and Health. Vol. 27. No. 1. 3-6.

[32] Kow, Y.M., Nardi, B. (2009) Culture and Creativity: World of Warcraft Modding in China and the US. Online Worlds: Convergence of the Real and the Virtual / Bainbridge W. (eds). Human-Computer Interaction Series. Berlin: Springer. P. 21-41.

[33] Smirnova E.O., Radeva, R.E. (2000) Psihologicheskie osobennosti kompyuternyh igr: novyj kontekst detskoj subkultury [Psychological features of computer games: a new context of the children's subculture]. Obrazovanie i informacionnaya kultura. Sociologicheskie aspekty [Education and informational culture. Sociological aspects]. Moscow: Centr sociologii obrazovaniya RAO Publ. pp. 330-336.

[34] Bányai, F., Griffiths, M. D., Király, O., Demetrovics, Z. (2018). The Psychology of Esports: A Systematic Literature Review. Journal of Gambling Studies. doi:10.1007/s10899-018-9763-1

[35] Kholodnaya, M.A. (2004) Kognitivnye stili: O prirode individual'nogo uma. [Cognitive styles: On the nature of the individual mind]. St. Petersburg: Piter Publ., 384 p. 